Article

\title{
Effect of Functional Group Density of Anion Exchange Resins on Removal of $p$-Toluene Sulfonic Acid from Aqueous Solution
}

\author{
Yue Sun ${ }^{1, * \mathbb{D}}$, Xiao Li ${ }^{1}$, Weisheng Zheng ${ }^{1}$, Xinchun Ding ${ }^{2}$ and Rajendra Prasad Singh ${ }^{1}$ \\ 1 Department of Municipal Engineering, School of Civil Engineering, Southeast University, \\ Nanjing 211189, China; 15720600151@163.com (X.L.); zhengwilson@163.com (W.Z.); \\ rajupsc@seu.edu.cn (R.P.S.) \\ 2 Nanjing University \& Yancheng Academy of Environmental Protection Technology and Engineering, \\ Yancheng 224051, China; wugao876@126.com \\ * Correspondence: sycyseu@163.com; Tel.: +86-25-83790750
}

Received: 11 November 2019; Accepted: 14 December 2019; Published: 18 December 2019

Featured Application: Five resins with different functional group density (SD1-SD5) possess an advantage in rapid adsorption equilibrium and high adsorption capacity. Additionally, they have better salt resistance with high levels of $\mathrm{Na}_{2} \mathrm{SO}_{4}$ in aromatic sulfonic acid (ASAs) wastewater.

\begin{abstract}
Adsorption using anion exchange resins is an efficient method for the removal of aromatic sulfonic acids (ASAs) from industrial wastewater. In this study, a series of weak-base anion exchangers (SD1-SD5) were synthesized to investigate the effect of functional group density of resins on the adsorption of ASAs from wastewater containing competitive inorganic anions. $p$-Toluene sulfonic acid (PTSA) was selected as a target pollutant, and $\mathrm{Na}_{2} \mathrm{SO}_{4}$ was chosen as the competitive inorganic salt because of its widespread existence in industrial wastewater. Adsorption performances of these resins were evaluated and compared in terms of selectivity, kinetics, isotherms, regeneration, and dynamic adsorption behavior. Importantly, the PTSA uptake increased with the raising content of functional groups on resins in the absence of $\mathrm{Na}_{2} \mathrm{SO}_{4}$; however, in the presence of a high level of $\mathrm{Na}_{2} \mathrm{SO}_{4}$ (for example, $\geq 1 \%$ ), a decrease in the functional group density could improve the adsorption capacity of resins for PTSA. Moreover, desorption and fixed bed column experiments were conducted in all resins, thereby confirming the effect of functional group density of resins on the PTSA adsorption in actual application. In brief, this research will provide a better understanding for the design and preparation of anion exchangers for the effective removal of ASA from wastewater.
\end{abstract}

Keywords: functional group density; $p$-toluene sulfonic acid; anion exchange resin; selectivity

\section{Introduction}

Water contamination by industrial organic effluent has attracted incessant attention all over the world. Aromatic sulfonic acids (ASAs), which are widely used as industrial intermediates and employed to synthetize acid compounds, dyes, pharmaceuticals, leather, and insecticides [1], are of significant concern because of their high water solubility and potential of causing severe damage to humans and the environment [2]. Therefore, it is crucial to effectively treat the wastewater from the manufacturing and subsequent using process of ASAs.

Many techniques have been adapted and proposed to deal with the industrial wastewater containing ASAs, such as advanced oxidation technology [3-5], biological treatment [6-8], extraction $[9,10]$, and adsorption $[2,11,12]$. Among these methods, adsorption is generally considered an effective process to remove organic pollutants and purify contaminated water due to its simplicity, 
rapidity, and low cost. Therefore, many adsorbents such as activated carbon, silica, modified fiber, chitosan, and polymeric resin have been used for wastewater treatment [13-17].

Among these available adsorbents, strong-base anion exchangers display better adsorption ability for ASAs owing to their strong electrostatic interaction, but their regeneration is an inherent challenge. Compared with strong-base anion exchange resin, weak-base anion exchangers show better regeneration performance, and some commercial weak-base anion exchangers such as D301 and ND900 have been widely applied for wastewater purification [2,18]. However, the results were not always satisfactory because of lower selectivity of these commercial resins for ASAs to inorganic anions, especially sulfate, which is often present in ASA waste streams at a high level $[19,20]$. Therefore, many efforts have been made by scientists to modify weak-base anion exchangers to improve their adsorption capacity and selectivity, mainly focusing on the optimization of functional groups [11,12,21-23] and the adjustment of the resin matrix structure [24]. It is worth noting that the functional group density of an adsorbent also affects its adsorption selectivity $[25,26]$. Moreover, the control of the number of functional groups is easy and less expensive than conventional modification operations. Nevertheless, few studies with regard to the functional group density of a weak-base anion exchanger affecting selective adsorption of ASAs from saline effluent have been reported so far.

Therefore, the objective of this paper is to prepare a series of weak-base anion exchangers with different functional group densities by controlling the dosage of dimethylamine as a functional reagent on chloromethylated polystyrene (CMPS). Afterwards, the synthesized resins were used for the removal of ASAs from aqueous solution with or without inorganic salts. $p$-Toluene sulfonic acid (PTSA) was selected as a representative of ASAs because of its toxicity and low biodegradability, and $\mathrm{Na}_{2} \mathrm{SO}_{4}$ as the competitive inorganic salt due to its wide existence in ASA wastewater. Batch adsorption experiments were carried out to evaluate the adsorption capacity and selectivity for PTSA for the sulfate of each resin. Taking into account practical application, column adsorption experiments were implemented using simulated industrial wastewater with PTSA and $\mathrm{Na}_{2} \mathrm{SO}_{4}$. The results would improve the understanding on the functional group density effects of weak-base anion exchange resins on removal of ASAs from saline wastewater and provide more choices for the design and optimization of anion exchangers used in a complex water environment.

\section{Materials and Methods}

\subsection{Materials}

CMPS (chlorine content 18.6\%, cross-linking density $6 \%$ ) was purchased from Zhengguang Co., Ltd. (Hangzhou, China). PTSA was purchased from Aldrich Sigma (Shanghai, China). Dimethylamine and other chemical reagents were purchased from Nanjing Chemical Reagent Co., Ltd. (Nanjing, China). All the chemicals were of analytical grade and used without further purification.

\subsection{Resin Synthesis and Characterization}

The resin synthesis was carried out in a $250 \mathrm{~mL}$ three-necked flask. To start the experiment, $30 \mathrm{~g}$ CMPS beads were swelled in $100 \mathrm{~mL}$ of $\mathrm{N}, \mathrm{N}$-dimethylformamide (DMF) for $8 \mathrm{~h}$ at room temperature under mechanical stirring at $160 \mathrm{rpm}$, and then filtered out of the suspension. Afterwards, $200 \mathrm{~mL}$ of dimethylamine solution was gradually introduced to a container containing the resin particles, and the mixture was stirred for $10 \mathrm{~h}$ at $313 \mathrm{~K}$. In the above amination process, the concentrations of dimethylamine solutions ranged from $10 \%$ to $40 \%$ (wt.) in order to obtain weak-base anion exchangers with different functional group densities. Finally, five resins (SD1-SD5) were obtained through the draining of residual dimethylamine solutions. The pictorial representation of the preparation procedures is shown in Scheme 1. 


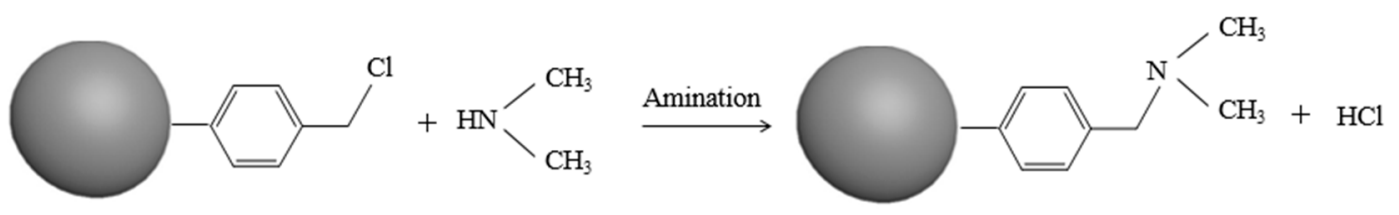

Scheme 1. Preparation route of resins.

Prior to use, all the resins were washed successively with $1 \mathrm{M} \mathrm{HCl}$, distilled water, and $1 \mathrm{M} \mathrm{NaOH}$, and then flushed to neutral $\mathrm{pH}$ with distilled water. Finally, the resins were extracted with absolute ethanol in a Soxhlet extractor for $8 \mathrm{~h}$ and vacuum dried at $313 \mathrm{~K}$ to constant weight. The surface area and pore size distribution of the resins were determined by the nitrogen adsorption and desorption method with a Micromeritics ASAP 2020 M automatic analyzer (USA). Infrared spectra analysis of CMPS and the tested resins was carried out by Nicolet 5700 Fourier transform infrared spectroscopy (FT-IR) (Madison, WI, USA) at ambient temperature. The X-ray photoelectron spectroscopy (XPS) analysis of the resins before and after adsorption was conducted by an ESCALAB-2 XPS spectrometer (UK).

\subsection{Batch Sorption Experiments}

Equilibrium adsorption of PTSA onto the resin was conducted in a conical flask containing a $100 \mathrm{~mL}$ solution shaken with $0.1 \mathrm{~g}$ resin, and the initial concentration range of PTSA was set to 200-1200 mg/L. $1 \mathrm{M} \mathrm{H}_{2} \mathrm{SO}_{4}$ or $\mathrm{NaOH}$ was used to adjust the solution $\mathrm{pH}$, and $\mathrm{Na}_{2} \mathrm{SO}_{4}$ was added into the solution to investigate the effect of coexisting anions. The flasks were then sealed and placed in a constant temperature shaker at a pre-settled temperature and $140 \mathrm{rpm}$ for $24 \mathrm{~h}$ to ensure the adsorption process had reached equilibrium. The equilibrium concentration of PTSA was measured spectrophotometrically at $261 \mathrm{~nm}$ by using a UV-vis spectrophotometer (Shanghai Jinghua Technology Instrument, China). The equilibrium adsorption capacity was calculated by conducting a mass balance on the PTSA before and after adsorption.

For the kinetic study, $0.3 \mathrm{~g}$ of each resin and $300 \mathrm{~mL}$ of PTSA solution with an initial content of $1000 \mathrm{mg} / \mathrm{L}$ were introduced into a $1000 \mathrm{~mL}$ flask quickly and shaken at $140 \mathrm{rpm}$ and $303 \mathrm{~K}$ continuously. Moreover, a $0.2 \mathrm{~mL}$ solution sample was taken out from the flask at different time intervals to determine adsorption kinetics.

\subsection{Desorption Experiments}

PTSA solution (100 mL of $1000 \mathrm{mg} / \mathrm{L}$ ) was introduced into $150 \mathrm{~mL}$ conical flasks and shaken with $0.1 \mathrm{~g}$ of each resin for $24 \mathrm{~h}$. After adsorption, the resin beads were transferred to another flask after filtration. A $100 \mathrm{~mL}$ mixed solution of $8 \% \mathrm{NaOH}$ and $5 \% \mathrm{NaCl}$ was added to each conical flask. Then, flasks were sealed and placed in a constant temperature shaker at $140 \mathrm{rpm}$ and $323 \mathrm{~K}$ for $24 \mathrm{~h}$ for resin regeneration. Finally, the amounts of PTSA eluted were evaluated.

\subsection{Column Sorption Experiments}

Column experiments were carried out with a glass column (18 $\mathrm{mm}$ diameter and $150 \mathrm{~mm}$ length) and the wet resin columns (SD1-SD5) were measured to be $10 \mathrm{~mL}$ each. The simulated solution containing $3700 \mathrm{mg} / \mathrm{L}$ PTSA and $3.4 \% \mathrm{Na}_{2} \mathrm{SO}_{4}$ was prepared by dissolving corresponding solutes in the deionized water, as was prescribed by Zhang [27]. The model solution passed through the resin column at a constant flow rate using a peristaltic pump. In addition, a water bath was equipped to maintain a constant temperature at $303 \mathrm{~K}$. All column runs were performed under the same hydrodynamic conditions: the superficial liquid velocity (SLV) was $0.08 \mathrm{~m} / \mathrm{h}$ and the empty bed contact time (EBCT) was $30 \mathrm{~min}$. 


\section{Results and Discussion}

\subsection{Characterization of Different Functional Group Density Resins}

As presented in Figure 1, all the resins were estimated to possess relatively wide pore size distributions and be rich in the macro and mesoporous region, which could enable adsorbate to reach the internal functional groups easily [28]. The basic parameters of the five resins are listed in Table 1. The BET surface area and pore volume decreased with the increase of functional group density, which was in line with the general findings from the preparation of porous anion exchangers by surface functionalization $[29,30]$.

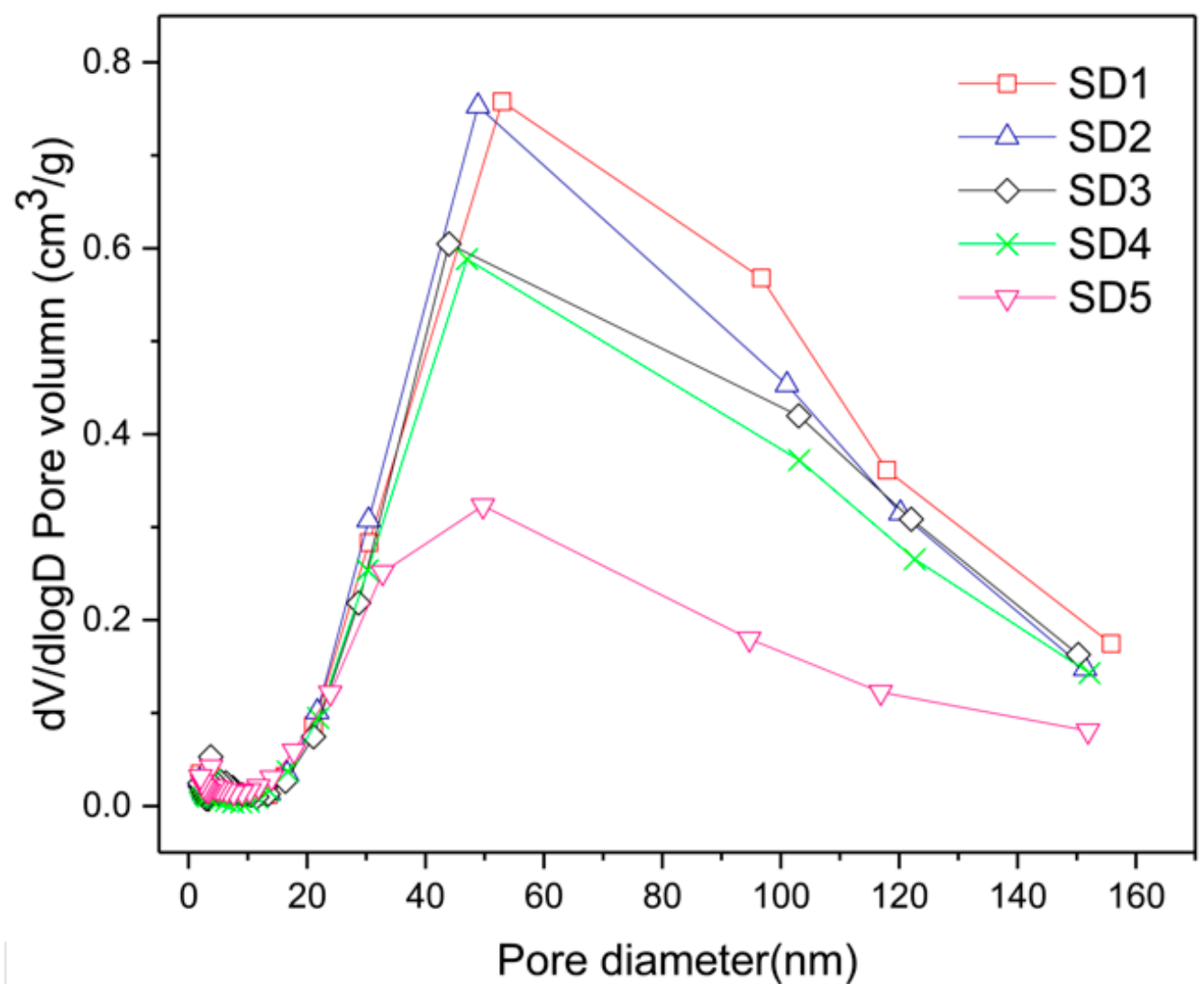

Figure 1. Pore size distribution of different resins.

Table 1. Basic parameters of different functional group density resins.

\begin{tabular}{cccccc}
\hline Resins & SD1 & SD2 & SD3 & SD4 & SD5 \\
\hline BET surface area $\left(\mathrm{m}^{2} / \mathrm{g}\right)$ & 50.43 & 49.01 & 47.45 & 42.17 & 37.96 \\
Pore volume $\left(\mathrm{cm}^{3} / \mathrm{g}\right)$ & 0.45 & 0.43 & 0.39 & 0.36 & 0.22 \\
Mean pore diameter $(\mathrm{nm})$ & 34.66 & 35.97 & 32.70 & 34.47 & 23.64 \\
Total exchange capacity $(\mathrm{mmol} / \mathrm{g})$ & 1.38 & 1.81 & 2.12 & 2.57 & 3.27 \\
Weakly basic exchange capacity $(\mathrm{mmol} / \mathrm{g})$ & 1.31 & 1.74 & 2.03 & 2.42 & 3.08 \\
Strongly basic exchange capacity $(\mathrm{mmol} / \mathrm{g})$ & 0.07 & 0.07 & 0.09 & 0.15 & 0.19 \\
\hline
\end{tabular}

Figure 2 represents the FT-IR spectra of the prepared resins and CMPS. In the CMPS spectra, the strong absorption peak at $673 \mathrm{~cm}^{-1}$ indicated that the CMPS contained quite a few C-Cl groups [31]. However, after amination reaction, this band gradually weakened or disappeared with the increase of the functional group densities of resins, which suggested that the chlorine methyl was involved in the reaction. Furthermore, all the synthetic resins showed strong C-N [32] and nitrogen methyl group [33] absorption bands at 1018 and $2776 \mathrm{~cm}^{-1}$, respectively [32]. Both the characteristic bands gradually 
increased with the increase of functional group density, implying that the dimethylamine functional group had been successfully grafted on the CMPS.

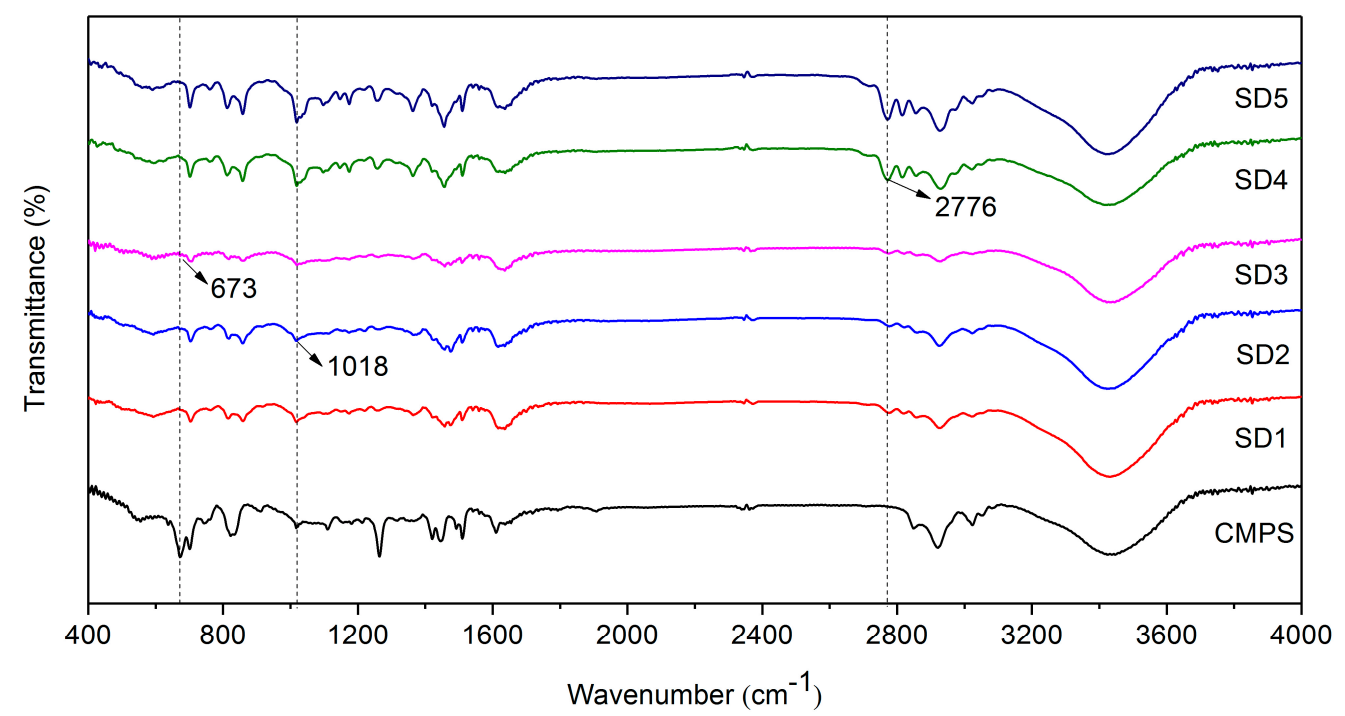

Figure 2. Fourier transform infrared spectroscopy (FT-IR) spectra of chloromethylated polystyrene (CMPS) and synthetic resins.

\subsection{Effect of $\mathrm{pH}$ on Adsorption}

Figure 3 illustrates the effect of solution $\mathrm{pH}$ on PTSA adsorption onto resins. The maximum PTSA uptake of each resin was observed when the $\mathrm{pH}$ value was about 2.3, which could be owing to the electrostatic interaction between the negatively charged PTSA and protonated amine groups on resins. With the solution $\mathrm{pH}$ value further increasing, even though the anionic species of PTSA increased $\left(\mathrm{p} K_{a}=1.7\right.$ [34]), the degree of protonation of amino groups on resins decreased, which was not conducive to electrostatic attraction. On the contrary, when the $\mathrm{pH}$ value was $<2.3$, with the $\mathrm{pH}$ value decreasing, protonation of amino groups on resins increased, but the neutral molecule form of PTSA increased, which could hinder adsorption though electrostatic interaction. Additionally, lower adsorption capacity in strong acidic solutions might also contribute to the introduced sulfate anion from $\mathrm{pH}$ adjustment. Sulfate anion could also be loaded onto resins by electrostatic interaction and result in the competitive adsorption with the PTSA anion [20].

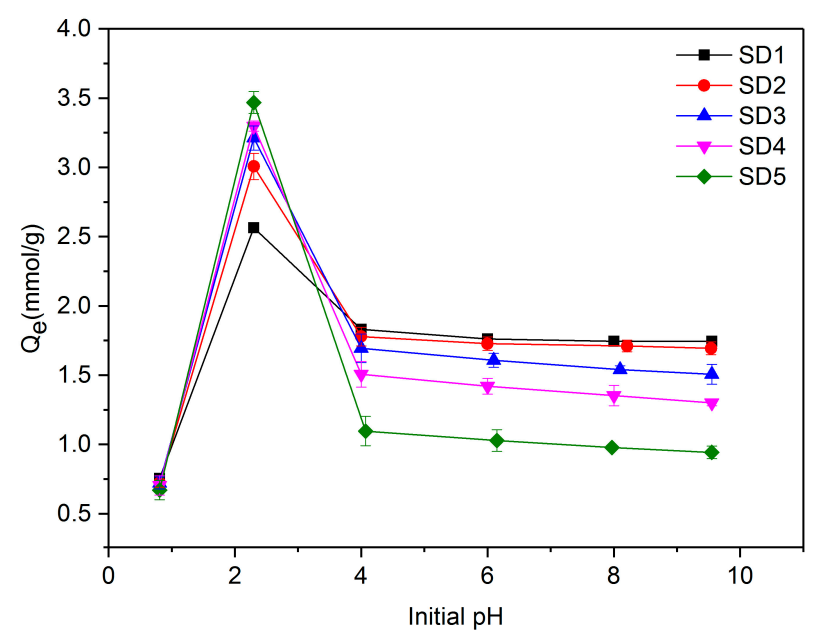

Figure 3. Effect of five resins on the $\mathrm{pH}$ adsorption of $p$-toluene sulfonic acid (PTSA). 


\subsection{Effect of $\mathrm{SO}_{4}{ }^{2-}$ on Adsorption and Selectivity of PTSA}

The selective adsorption property of an exchanger towards ASAs is of particular significance for its future application because inorganic anions often coexist with ASAs at a high level in the industrial waste streams. Excessive inorganic anions may interfere with the adsorption of ASAs on the resin due to the competitive effect. Thus, $\mathrm{Na}_{2} \mathrm{SO}_{4}$ was selected as a typical inorganic salt due to its widespread coexistence in ASA chemical effluents. Figure 4 shows the influence of coexisting $\mathrm{Na}_{2} \mathrm{SO}_{4}$ on PTSA adsorption by the five resins from water.

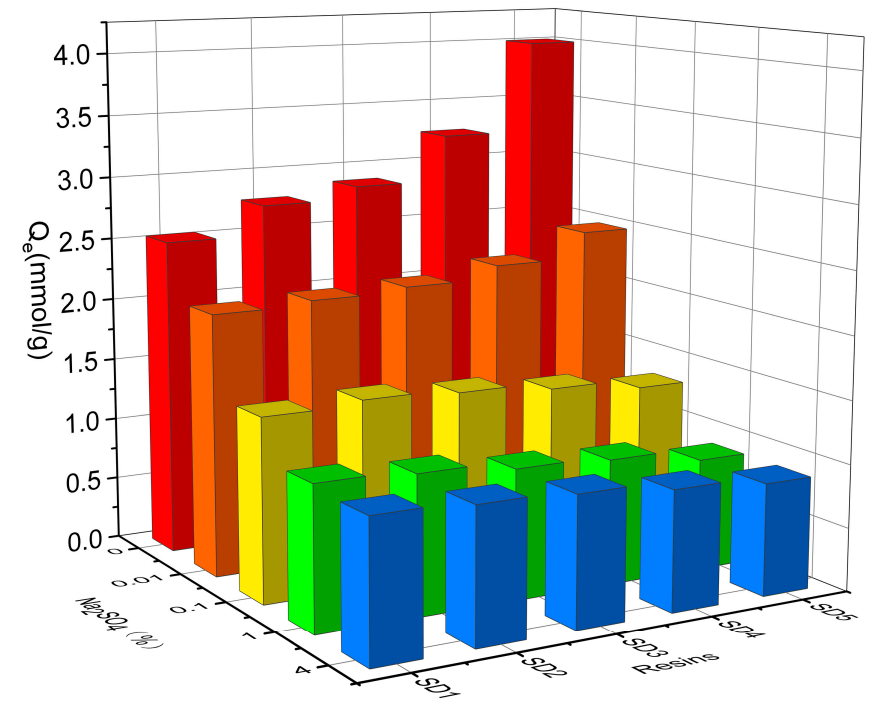

Figure 4. Effect of $\mathrm{Na}_{2} \mathrm{SO}_{4}$ concentration on PTSA adsorption by different resins.

In the absence of $\mathrm{Na}_{2} \mathrm{SO}_{4}$, the PTSA uptake increased in the order of SD1 $<\mathrm{SD} 2<\mathrm{SD} 3<\mathrm{SD} 4<$ $\mathrm{SD} 5$, which agreed with the functional group density of resins. Hence, the higher the functional group density of a resin, the higher the PTSA adsorption capacity. Nevertheless, the adsorption amount of resins significantly decreased with increase in the initial concentration of $\mathrm{Na}_{2} \mathrm{SO}_{4}$, suggesting that there was a strong competitive effect between the sulfate radical and the PTSA anion [24]. When the $\mathrm{Na}_{2} \mathrm{SO}_{4}$ content in the solution increased to $1 \%$, it was found that the adsorption amount order completely reversed to $\mathrm{SD} 5<\mathrm{SD} 4<\mathrm{SD} 3<\mathrm{SD} 2<\mathrm{SD} 1$. In other words, reducing the functional group density of resins could improve the adsorption selectivity of resins toward PTSA in the presence of a competing sulfate anion.

In general, there are two main mechanisms involved in the adsorption of ASAs on a polymeric anion exchanger, i.e., hydrophobic interaction and electrostatic interaction $[35,36]$. The resin with a lower functional group density possessed the higher hydrophobicity, which was conducive to hydrophobic interaction between resin phase and PTSA rather than sulfate. Another possible reason for this observation could be ascribed to the changing distance between functional groups of resin surface. When the two adjacent active sites were far apart resulting from the decrease of functional group density, they would be unlikely to interact with a single divalent anion such as sulfate. PTSA anion is monovalent, a trait which was unaffected by the distance between functional groups on the resin.

To further quantitatively compare the selectivity of different resins, binary competition tests were conducted. The distribution ratio $K_{d}(\mathrm{~mL} / \mathrm{g})$ and the selectivity coefficient $\alpha$ were calculated by Equation (1) [37] and Equation (2) [38], respectively.

$$
k_{d}=\frac{Q_{e}}{C_{e}}
$$




$$
\alpha=\frac{K_{d 1}}{K_{d 2}}
$$

where $C_{e}$ is the equilibrium concentration of adsorbate in solution; $Q_{e}$ is the equilibrium adsorption capacity of adsorbent; $K_{d 1}$ and $K_{d 2}$ represent the distribution ratios of PTSA (target adsorbate) and sulfate (competitive adsorbate) on resins, respectively; and $\alpha$ represents the relative affinity of the adsorbent for PTSA with respect to sulfate.

It can be observed that the $\alpha$ values of five resins sequentially decreased in the order of SD1 > SD2 > SD3 > SD4 > SD5 under the three different initial concentrations in Table 2, signifying that the salt resistance property of all resins decreased in the same order. In addition, the values of the selectivity coefficient $\alpha$ of the resins decreased continuously when the concentrations of PTSA and sulfate increased, owing to the intensified competition between PTSA and sulfate.

Table 2. Selectivity coefficient $\alpha$ of PTSA and $\mathrm{SO}_{4}{ }^{2-}$ adsorption on resins in a binary system.

\begin{tabular}{cccc}
\hline \multirow{2}{*}{ Adsorbents } & \multicolumn{3}{c}{ Initial Concentration of PTSA $\left(\mathbf{m g} / \mathrm{L}^{2} / \mathbf{S O}_{\mathbf{4}}{ }^{\mathbf{2 -}} \mathbf{( \mathbf { m g } / \mathrm { L } )}\right.$} \\
\cline { 2 - 4 } & $\mathbf{1 0 0 / 1 0 0}$ & $\mathbf{5 0 0 / 5 0 0}$ & $\mathbf{1 0 0 0 / 1 0 0 0}$ \\
\hline SD1 & 3.2010 & 0.8078 & 0.5074 \\
SD2 & 2.9223 & 0.7786 & 0.4628 \\
SD3 & 2.3752 & 0.6774 & 0.4014 \\
SD4 & 2.1478 & 0.6490 & 0.3455 \\
SD5 & 1.3690 & 0.5812 & 0.3003 \\
\hline
\end{tabular}

\subsection{Adsorption Kinetics of PTSA}

Adsorption kinetics is significant in the study of the performance of adsorbents for practical application. The kinetic curves of PTSA adsorption on five resins are displayed in Figure 5. To further evaluate adsorption behavior, the data were analyzed using the pseudo-first-order and pseudo-second-order kinetic models (Equations (3) and (4) respectively).

$$
\begin{gathered}
Q_{t}=Q_{e}\left(1-e^{-k 1 t}\right) \\
Q_{t}=\frac{k_{2} Q_{e}^{2} t}{1+k_{2} Q_{e} t}
\end{gathered}
$$

where $Q_{e}$ and $Q_{t}(\mathrm{mmol} / \mathrm{g})$ are the adsorption capacities of PTSA at equilibrium and at time $t(\mathrm{~min})$, respectively; and $k_{1}(1 / \mathrm{min})$ and $k_{2}(\mathrm{~g} /(\mathrm{mmol} \cdot \mathrm{min}))$ are the rate constants of the pseudo-first-order and pseudo-second-order kinetic models, respectively. The calculated correlation parameters are presented in Table 3.

As can be seen from Table 3, the values of the calculated adsorption capacities $Q_{e, c a l}$ were much closer to the experimental uptake $Q_{e, e x p}$, and the higher values of $R^{2}$ suggested that the pseudo-second-order model could better explain the adsorption process. The rate constants $k_{2}$ for PTSA adsorption onto SD1, SD2, and SD3 resins were not significantly different, but significantly larger than SD4 and SD5, which might be related to the surface area and pore structure of the resins. According to Table 1, the BET surface area and pore volume of the five resins are in the following order: SD1 $>$ SD2 $>$ SD3 $>$ SD4 $>$ SD5. Hence, the resins with larger pore volume and specific surface area can accelerate the continuous reaction between adsorbent and PTSA molecules. Additionally, in water, the amino groups on resins would stretch due to their hydrophilic property, which could result in the obstruction of the effective pore canal for the adsorbate diffusion [39]. Thus, a slower PTSA adsorption rate reasonably occurred in the resins with higher functional group density, such as SD4 and SD5. 


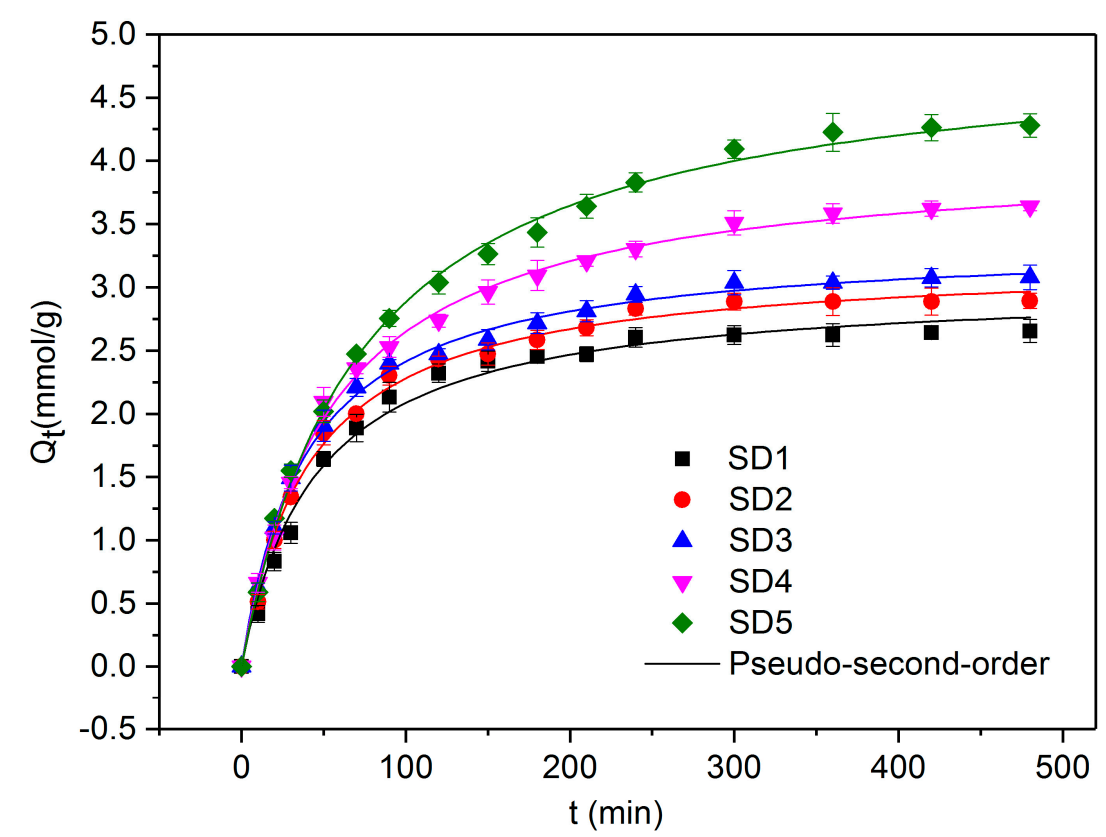

Figure 5. Adsorption kinetics of PTSA onto resins at $303 \mathrm{~K}$.

Table 3. Kinetic data for the adsorption of PTSA onto resins.

\begin{tabular}{|c|c|c|c|c|c|c|c|}
\hline \multirow{3}{*}{ Adsorbents } & \multirow{3}{*}{$\begin{array}{c}Q_{e, \exp } \\
(\mathrm{mmol} / \mathrm{g})\end{array}$} & \multicolumn{3}{|c|}{ Pseudo-First-Order } & \multicolumn{3}{|c|}{ Pseudo-Second-Order } \\
\hline & & $Q_{e, c a l}$ & $k_{1}$ & \multirow{2}{*}{$R^{2}$} & $Q_{e, c a l}$ & $k_{2}$ & \multirow{2}{*}{$R^{2}$} \\
\hline & & $(\mathrm{mmol} / \mathrm{g})$ & $(1 / \mathrm{min})$ & & $(\mathrm{mmol} / \mathrm{g})$ & $(\mathrm{g} / \mathrm{mmol} \cdot \mathrm{min})$ & \\
\hline SD1 & 2.8419 & 2.6114 & 0.0184 & 0.9976 & 3.0208 & 0.0073 & 0.9888 \\
\hline SD2 & 3.0869 & 2.8045 & 0.0195 & 0.9883 & 3.2218 & 0.0075 & 0.9952 \\
\hline SD3 & 3.2943 & 2.9373 & 0.0202 & 0.9819 & 3.3630 & 0.0075 & 0.9974 \\
\hline SD4 & 3.8598 & 3.4670 & 0.0157 & 0.9828 & 4.0619 & 0.0046 & 0.9975 \\
\hline SD5 & 4.3795 & 4.1325 & 0.0123 & 0.9838 & 4.5570 & 0.0028 & 0.9975 \\
\hline
\end{tabular}

\subsection{Adsorption Isotherm of PTSA}

The adsorption isotherms of PTSA on resins at $303 \mathrm{~K}$ are depicted in Figure 6. The adsorption amounts of all the resins increased with the rise of initial PTSA concentration. To further evaluate adsorption behavior, the data were analyzed using the Langmuir (Equation (5)) and Freundlich (Equation (6)) equilibrium models.

$$
\begin{gathered}
Q_{e}=\frac{K_{L} \times Q_{m} \times C_{e}}{1+K_{L} \times C_{e}} \\
Q_{e}=K_{F} \times C_{e}^{\frac{1}{n}}
\end{gathered}
$$

where $C_{e}(\mathrm{mmol} / \mathrm{L})$ is the equilibrium concentration of PTSA; $Q_{e}(\mathrm{mmol} / \mathrm{g})$ represents the equilibrium adsorption capacity of the resin; and $K_{L}(\mathrm{~L} / \mathrm{mmol}), Q_{m}(\mathrm{mmol} / \mathrm{g}), K_{F}(\mathrm{~L} / \mathrm{g})$, and $n$ are the characteristic constants. As listed in Table 4, the adsorption isotherms were well described by both the Langmuir and Freundlich models due to the high values of correlation coefficients $\left(R^{2}\right)$. The value of $\mathrm{n}$ was greater than unity, indicating that the PTSA was favorably adsorbed by the five resins. Thus, all the results denoted that the adsorption process may occur on heterogeneous surfaces, and that the adsorption is not restricted to monolayer formation [8]. Both $Q_{m}$ and $K_{F}$, which are relative to the adsorption capacity in Langmuir and Freundlich models, respectively, decreased in the order of SD5 > SD4 > SD3 > SD2 > SD1. This was consistent with the result in Section 3.3, when there was no sulfate competition system. 


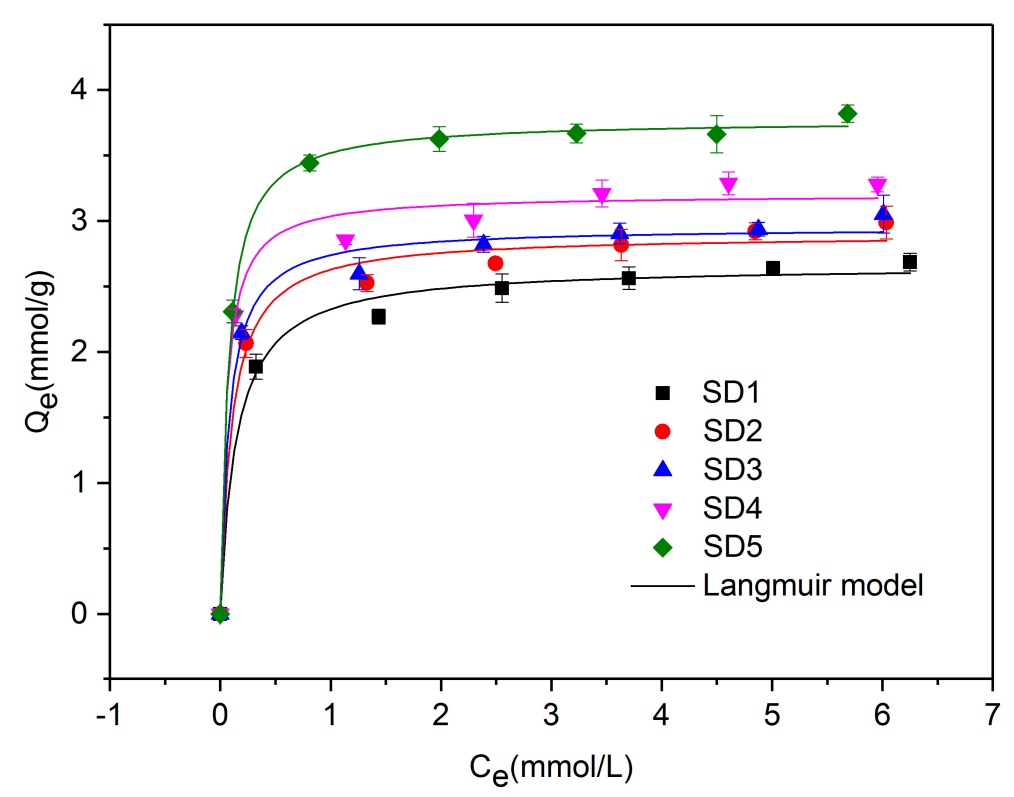

Figure 6. Adsorption isotherms of PTSA onto resins at $303 \mathrm{~K}$.

Table 4. Characteristic properties of adsorption isotherms.

\begin{tabular}{ccccccc}
\hline \multirow{2}{*}{ Adsorbents } & \multicolumn{3}{c}{ Langmuir } & \multicolumn{3}{c}{ Freundlich } \\
\cline { 2 - 7 } & $\boldsymbol{Q}_{\boldsymbol{m}} \mathbf{( \mathbf { m m o l } / \mathbf { g } )}$ & $\boldsymbol{K}_{\boldsymbol{L}}(\mathbf{L} / \mathbf{m m o l})$ & $\boldsymbol{R}^{\mathbf{2}}$ & $\boldsymbol{K}_{\boldsymbol{F}}$ & $\boldsymbol{n}$ & $\boldsymbol{R}^{\mathbf{2}}$ \\
\hline SD1 & 2.7589 & 9.5222 & 0.9906 & 2.3169 & 8.4754 & 0.9972 \\
SD2 & 2.9988 & 11.1786 & 0.9920 & 2.5355 & 8.3759 & 0.9982 \\
SD3 & 3.2578 & 9.6817 & 0.9925 & 2.7110 & 7.5409 & 0.9928 \\
SD4 & 3.4916 & 13.3726 & 0.9935 & 2.9623 & 7.9107 & 0.9930 \\
SD5 & 4.2401 & 13.1659 & 0.9939 & 3.5859 & 6.9177 & 0.9804 \\
\hline
\end{tabular}

\subsection{XPS Analysis before and after Adsorption}

For further characterization of the resins before and after adsorption, The XPS spectra of SD1 and SD5 before and after adsorption are exhibited in Figure 7. As seen, all S2p spectra of the two resins before adsorption were not detected to any peaks. Nonetheless, the S2p spectra of SD1 and SD5 were revealed at the increased peak of 168.18 and $168.00 \mathrm{eV}$, respectively, after adsorption, which illustrated that sulfur (sulfonic acid groups in PTSA) was adsorbed on the surface of the adsorbents $[16,40]$.

As seen in Figure 7c, before adsorption, three N1s spectra of SD1 resin peaks were detected, which were assigned as either free tertiary amine groups $(399.55 \mathrm{eV})$, protonated tertiary amine groups (402.43 eV), or quaternary ammonium groups (403.35 eV) [41]. As seen from Table 1, all resins possessed a strong base exchange capacity according to the basic parameters of different functional group density resins, which demonstrates the presence of quaternary ammonium nitrogen N3 in the resin. Furthermore, it can be found that the structure of SD1 resin no longer contains the free tertiary amine group N1 after adsorption on the basis of the area ratio of different forms of nitrogen atoms after adsorption of PTSA. In addition, the area ratio of N1 in SD5 resin decreased from $66.63 \%$ to $10.14 \%$ after adsorption, as seen in Figure $7 \mathrm{~d}$. We speculate that this is due to the free tertiary amine group having been protonated and having participated in the electrostatic interaction between the resin and PTSA. In addition, the area ratio of protonated tertiary amine groups in the two resins after adsorption was $93.01 \%$ and $85.86 \%$, respectively, implying that the degree of protonation of the amination group may be related to the density of the functional group of the resin. Hence, the higher the functional group density, the weaker the conversion ability of free tertiary amine groups to protonated tertiary amine groups. 

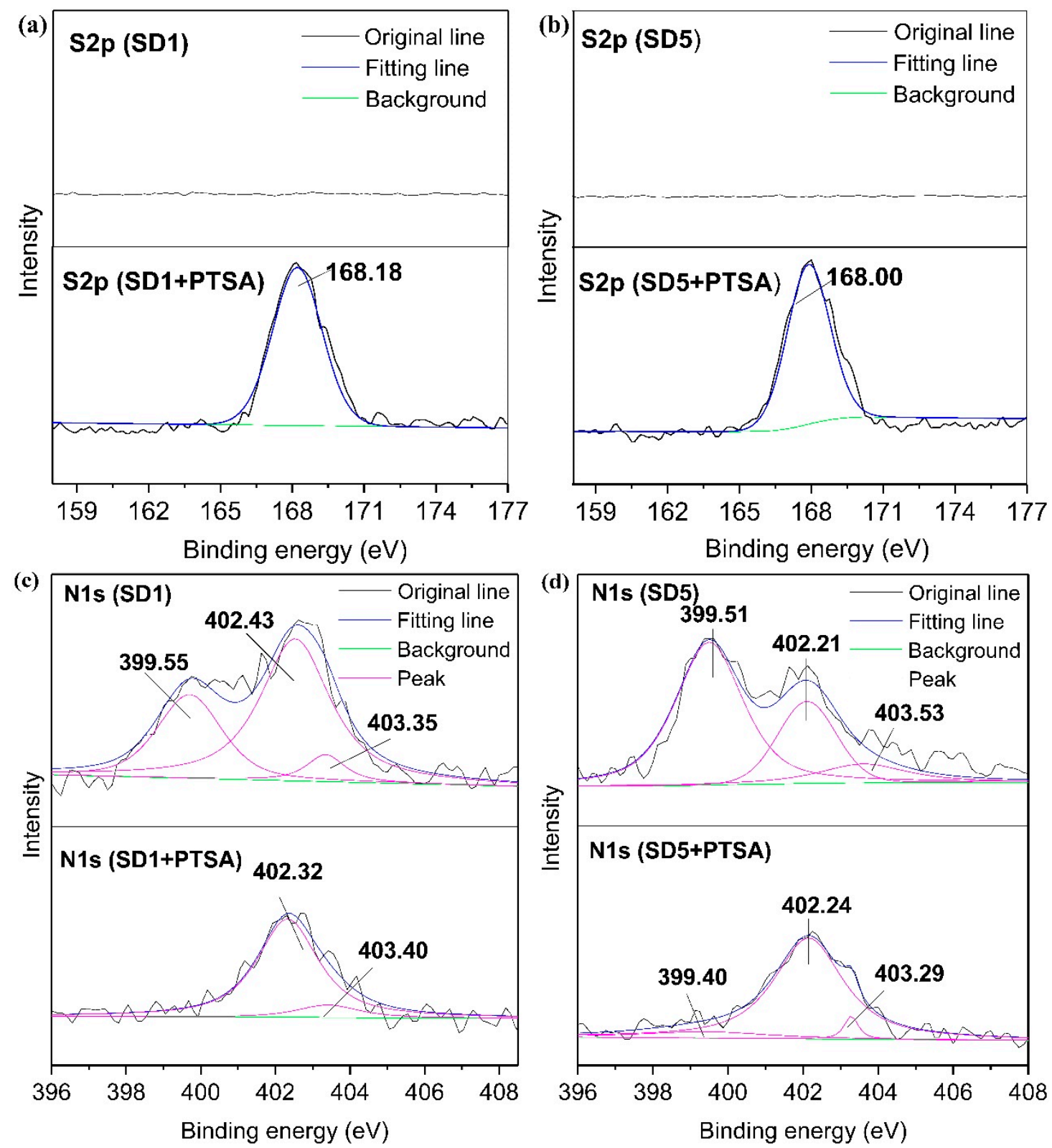

Figure 7. X-ray photoelectron spectroscopy (XPS) spectra of (a) S2p of SD1 resin, (b) S2p of SD5 resin, (c) N1s of SD1 resin and (d) N1s of SD5 resin before and after adsorption.

\subsection{Desorption}

Regeneration of saturated adsorbents is of vital significance for actual application. In view of the fact that $\mathrm{NaOH}$ can result in deprotonation of the fixed amine groups resin and $\mathrm{NaCl}$ can disturb electrostatic attraction between the net positively charged resin surface and PTSA anion, the mixed solution of $8 \% \mathrm{NaOH}$ and $5 \% \mathrm{NaCl}$ was chosen as the eluent, and the desorption efficiencies of five resins are presented in Figure 8. Notably, the final static desorption rate of each resin was above $80 \%$, hinting that the PTSA adsorption on the resins was reversible. Meanwhile, it clearly suggested that electrostatic interaction played a leading role in the PTSA adsorption on resins. Moreover, a decreasing trend in the functional group density of resins was favorable for the desorption performance of the resin (Figure 8). 


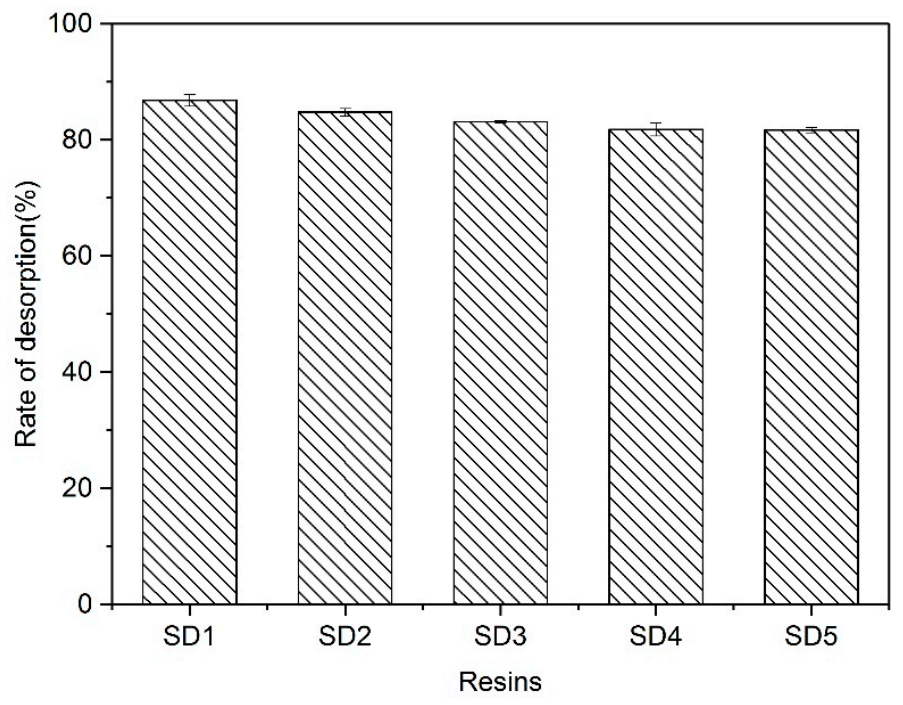

Figure 8. Static desorption of PTSA-loaded resins.

\subsection{Column Sorption Experiments}

To further assess the feasibility of resins in practical applications, dynamic experiments were performed to remove PTSA from the simulated wastewater containing $3700 \mathrm{mg} / \mathrm{L} \mathrm{PTSA}$ and $3.4 \%$ competing $\mathrm{Na}_{2} \mathrm{SO}_{4}$ [27], and the breakthrough curves are shown in Figure 9. The results revealed that the $10 \%$ breakthrough of PTSA occurred in 24 BV for SD5 resin, while such a breakthrough could be found in $30 \mathrm{BV}$ for the other resins. In comparison with SD5, the resins with relatively low functional group densities showed a more satisfactory dynamic adsorption property, which could be ascribed to their enhanced selectivity for PTSA.

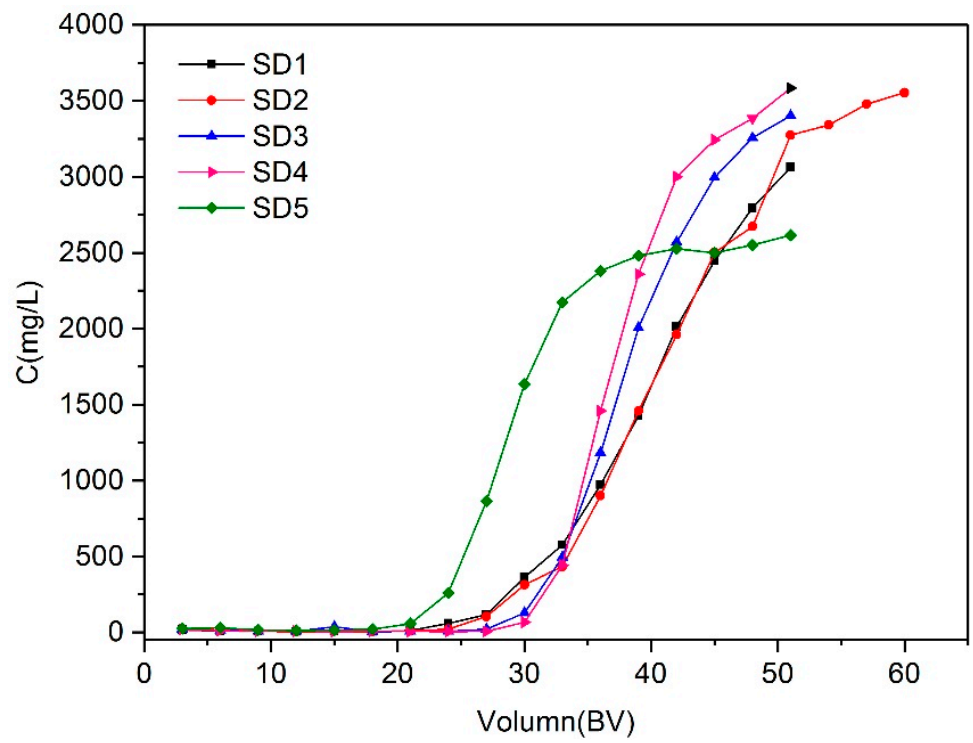

Figure 9. Breakthrough curves of PTSA adsorbed on resins.

\section{Conclusions}

In this study, a series of resins with different functional group densities were prepared and employed to investigate the adsorption behavior of PTSA. The adsorption capacity of resins in a salt-free system followed the order of SD5 $>\mathrm{SD} 4>\mathrm{SD} 3>\mathrm{SD} 2>\mathrm{SD} 1$, which agreed with the functional group density of the resins. When the $\mathrm{Na}_{2} \mathrm{SO}_{4}$ content in the solution increased to $1 \%$, the adsorption 
amount order completely reversed to SD5 $<$ SD4 $<$ SD3 $<$ SD2 $<$ SD1. More than $80 \%$ PTSA desorption rate was achieved from exhausted resins by using a mixed solution of $8 \% \mathrm{NaOH}$ and $5 \% \mathrm{NaCl}$. In addition, the results of the column experiment demonstrated that the resins with relatively low functional group densities had enhanced adsorption properties towards PTSA from the simulated wastewater containing $\mathrm{Na}_{2} \mathrm{SO}_{4}$. In summary, the present work could offer significant guidance for the design of weak-basic anion exchangers for the removal of PTSA and other ASAs from wastewaters with high concentration of competing $\mathrm{Na}_{2} \mathrm{SO}_{4}$.

Author Contributions: Conceptualization, Y.S.; Formal analysis, Y.S.; Funding acquisition, Y.S.; Resources, X.D.; Visualization, X.L.; Writing_original draft, Y.S.; Writing—review \& editing, X.L., W.Z. and R.P.S. All authors have read and agreed to the published version of the manuscript.

Funding: This research was funded by National Natural Science Foundation of China (No. 51578131) and the Open Foundation of the Institute of Water Environmental Engineering, Jiangsu Industrial Technology Research Institute, Yancheng (NDYCKF201701). The APC was funded by the National Natural Science Foundation of China (No. 51578131).

Conflicts of Interest: The authors declare no conflict of interest.

\section{References}

1. Ravera, M.; Buico, A.; Gosetti, F.; Cassino, C.; Musso, D.; Osella, D. Oxidative degradation of 1,5-naphthalenedisulfonic acid in aqueous solutions by microwave irradiation in the presence of $\mathrm{H}_{2} \mathrm{O}_{2}$. Chemosphere 2009, 74, 1309-1314. [CrossRef] [PubMed]

2. Pan, B.; Zhang, Q.; Meng, F.; Li, X.; Zhang, X.; Zheng, J.; Zhang, W.; Pan, B.; Chen, J. Sorption enhancement of aromatic sulfonates onto an aminated hyper-cross-linked polymer. Environ. Sci. Technol. 2005, 39, 3308-3313. [CrossRef] [PubMed]

3. Zhu, S.N.; Wang, C.; Yip, A.C.; Tsang, D.C. Highly effective degradation of sodium dodecylbenzene sulphonate and synthetic greywater by Fenton-like reaction over zerovalent iron-based catalyst. Environ. Technol. 2015, 36, 1423-1432. [CrossRef] [PubMed]

4. Olmezhanci, T.; Arslanalaton, I.; Gelegen, O. Photo-Fenton-like treatment of K-acid: Assessment of treatability, toxicity and oxidation products. Water Sci. Technol. 2014, 70, 1056-1064. [CrossRef] [PubMed]

5. Avetta, P.; Prevot, A.B.; Fabbri, D.; Montoneri, E.; Tomasso, L. Photodegradation of naphthalene sulfonic compounds in the presence of a bio-waste derived sensitizer. Chem. Eng. J. 2012, 197, 193-198. [CrossRef]

6. de Souza, N.A.; Ramaiah, N.; Damare, S.; Furtado, B.; Mohandass, C.; Patil, A.; de Lima, M. Differential Protein Expression in Shewanella seohaensis Decolorizing Azo Dyes. Curr. Proteom. 2019, 16, $156-164$. [CrossRef]

7. Song, Z.; Song, L.; Shao, Y.; Tan, L. Degradation and detoxification of azo dyes by a salt-tolerant yeast Cyberlindnera samutprakarnensis S4 under high-salt conditions. World J. Microb. Biot. 2018, 34, 131. [CrossRef]

8. Wan, S.; Hua, Z.; Sun, L.; Bai, X.; Liang, L. Biosorption of nitroimidazole antibiotics onto chemically modified porous biochar prepared by experimental design: Kinetics, thermodynamics, and equilibrium analysis. Process Saf. Environ. 2016, 104, 422-435. [CrossRef]

9. Liu, Y.; Xu, D.-L.; Wang, P.; Dong, Y.-H. Removal of sodium salts and chemical oxygen demand from real reactive dye wastewater by the integrated process of chemical precipitation and extraction. Desalin. Water Treat. 2015, 57, 6772-6780. [CrossRef]

10. Gai, H.; Zhong, C.; Qiao, L.; Chen, S.; Xiao, M.; Song, H. Extraction of 1-amino-2-Naphthol-4-Sulfonic acid from wastewater using trioctylamine (N, N-dioctyloctan-1-amine) in methyl isobutyl ketone. J. Clean. Prod. 2018, 201, 774-782. [CrossRef]

11. Huang, J.; Yang, L.; Zhang, Y.; Pan, C.; Liu, Y.-N. Resorcinol modified hypercrosslinked poly(styrene-co-divinlybenzene) resin and its adsorption equilibriums, kinetics and dynamics towards p-hydroxylbenzaldehyde from aqueous solution. Chem. Eng. J. 2013, 219, 238-244. [CrossRef]

12. de Abreu Domingos, R.; da Fonseca, F.V. Evaluation of adsorbent and ion exchange resins for removal of organic matter from petroleum refinery wastewaters aiming to increase water reuse. J. Environ. Manag. 2018, 214, 362-369. [CrossRef] [PubMed] 
13. Davarpanah, M.; Ahmadpour, A.; Rohani-Bastami, T.; Dabir, H. Synthesis and application of diethanolamine-functionalized polystyrene as a new sorbent for the removal of p-toluenesulfonic acid from aqueous solution. J. Ind. Eng. Chem. 2015, 30, 281-288. [CrossRef]

14. Jiang, C.; Wang, X.; Wang, G.; Hao, C.; Li, X.; Li, T. Adsorption performance of a polysaccharide composite hydrogel based on crosslinked glucan/chitosan for heavy metal ions. Compos. Part B Eng. 2019, 169, 45-54. [CrossRef]

15. Thitame, P.V.; Shukla, S.R. Adsorptive removal of naphthalenesulfonic acids using wild almond shell activated carbon from aqueous solution. Environ. Prog. Sustain. 2017, 36, 38-44. [CrossRef]

16. Zhang, Z.; Wang, F.; Yang, W.; Yang, Z.; Li, A. A comparative study on the adsorption of 8-amino-1-naphthol-3,6-disulfonic acid by a macroporous amination resin. Chem. Eng. J. 2016, 283, 1522-1533. [CrossRef]

17. Ali, I.; Alharbi, O.M.L.; Alothman, Z.A.; Al-Mohaimeed, A.M.; Alwarthan, A. Modeling of fenuron pesticide adsorption on CNTs for mechanistic insight and removal in water. Environ. Res. 2019, 170, 389-397. [CrossRef]

18. Zang, Y.; Yue, Q.; Kan, Y.; Zhang, L.; Gao, B. Research on adsorption of Cr(VI) by Poly-epichlorohydrin -dimethylamine (EPIDMA) modified weakly basic anion exchange resin D301. Ecotoxicol. Environ. Saf. 2018, 161, 467-473. [CrossRef]

19. Sun, Y.; Zuo, P.; Luo, J.; Singh, R.P. Adsorption behavior of benzenesulfonic acid by novel weakly basic anion exchange resins. J. Environ. Sci. 2017, 54, 40-47. [CrossRef]

20. Pan, B.; Zhang, Q.; Pan, B.; Zhang, W.; Du, W.; Ren, H. Removal of aromatic sulfonates from aqueous media by aminated polymeric sorbents: Concentration-dependent selectivity and the application. Microporous Mesoporous Mater. 2008, 116, 63-69. [CrossRef]

21. Lu, Z.; Jiang, B.; Li, A. Investigation of the anti-fouling performance of an aminated resin. Chem. Eng. J. 2012, 193-194, 139-145. [CrossRef]

22. Gokmen, M.T.; Prez, F.E.D. Porous polymer particles-A comprehensive guide to synthesis, characterization, functionalization and applications. Prog. Polym. Sci. 2012, 37, 365-405. [CrossRef]

23. Huang, J.; Jin, X.; Mao, J.; Yuan, B.; Deng, R.; Deng, S. Synthesis, characterization and adsorption properties of diethylenetriamine-modified hypercrosslinked resins for efficient removal of salicylic acid from aqueous solution. J. Hazard. Mater. 2012, 217-218, 406-415. [CrossRef] [PubMed]

24. Tan, L.; Shuang, C.; Wang, Y.; Wang, J.; Su, Y.; Li, A. Effect of pore structure on the removal of clofibric acid by magnetic anion exchange resin. Chemosphere 2018, 191, 817-824. [CrossRef] [PubMed]

25. Walcarius, A.; Delacôte, C. Mercury(II) binding to thiol-functionalized mesoporous silicas: Critical effect of $\mathrm{pH}$ and sorbent properties on capacity and selectivity. Anal. Chim. Acta 2005, 547, 3-13. [CrossRef]

26. Zhu, Y.; Zhou, J.; Hu, J.; Liu, H. The effect of grafted amine group on the adsorption of $\mathrm{CO}_{2}$ in $\mathrm{MCM}^{-41}$ : A molecular simulation. Catal. Today 2012, 194, 53-59. [CrossRef]

27. Ling, Z. Study on Wastewater Treatment and Resource Utilization of Doxycycline Production. Master's Thesis, Henan University, Kaifeng, Henan, 2006.

28. Deepatana, A.; Valix, M. Steric hindrance effect on adsorption of metal-organic complexes onto aminophosphonate chelating resin. Desalination 2008, 218, 297-303. [CrossRef]

29. Lee, B.; Lee, S.D.; Choo, K.-H. The removal of perchlorate using amine-functionalized mesoporous anion-exchange resins with different number of ligands. Korean J. Chem. Eng. 2013, 30, 898-905. [CrossRef]

30. Zhang, Q.; Pan, B.; Zhang, W.; Pan, B.; Zhang, Q.; Ren, H. Arsenate Removal from Aqueous Media by Nanosized Hydrated Ferric Oxide (HFO)-Loaded Polymeric Sorbents: Effect of HFO Loadings. Ind. Eng. Chem. Res. 2008, 47, 3957-3962. [CrossRef]

31. Zhao, H.C.; Guo, J.L.; Li, J.T.; Gao, L.L.; Bian, C.C. Synthesis and Thermal Property of Linear Chloromethylated Polystyrene. Adv. Mater. Res. 2010, 150-151, 1504-1507. [CrossRef]

32. Siddiqi, H.M.; Siraj, A.; Khalid, N.; Akhtar, Z.; Haq, M.Z.U. Thermally stable epoxy polymers from new tetraglycidyl amine-based resin. J. Therm. Anal. Calorim. 2018, 132, 205-214. [CrossRef]

33. Pan, B.C.; Xiong, Y.; Su, Q.; Li, A.M.; Chen, J.L.; Zhang, Q.X. Role of amination of a polymeric adsorbent on phenol adsorption from aqueous solution. Chemosphere 2003, 51, 953-962. [CrossRef]

34. Tawde, S.; Mukesh, D.; Yakhmi, J.V. Redox behavior of polyaniline as influenced by aromatic sulphonate anions: Cyclic voltammetry and molecular modeling. Synth. Met. 2001, 125, 401-413. [CrossRef] 
35. Pan, B.; Zhang, W.; Pan, B.; Qiu, H.; Zhang, Q.; Zhang, Q.; Zheng, S. Efficient removal of aromatic sulfonates from wastewater by a recyclable polymer: 2-naphthalene sulfonate as a representative pollutant. Environ. Sci. Technol. 2008, 42, 7411-7416. [CrossRef] [PubMed]

36. Yang, W.B.; Li, A.M.; Fu, C.; Fan, J.; Zhang, Q.X. Adsorption mechanism of aromatic sulfonates onto resins with different matrices. Ind. Eng. Chem. Res. 2007, 46, 6971-6977. [CrossRef]

37. Li, L.; Liu, F.; Jing, X.; Ling, P.; Li, A. Displacement mechanism of binary competitive adsorption for aqueous divalent metal ions onto a novel IDA-chelating resin: Isotherm and kinetic modeling. Water Res. 2011, 45, 1177-1188. [CrossRef]

38. Maity, N.; Payne, G.F.; Chipchosky, J.L. Adsorptive separations based on the differences in solute-sorbent hydrogen-bonding strengths. Ind. Eng. Chem. Res. 1991, 30, 2456-2463. [CrossRef]

39. Sun, Y.; Li, A.; Zhang, Q.; Chen, J.; Fu, D.; Wang, S. Adsorptive separation of tannic acid from aqueous solution by polymeric resins. Sep. Sci. Technol. 2008, 43, 389-402. [CrossRef]

40. Ji, C.; Song, S.; Wang, C.; Sun, C.; Qu, R.; Wang, C.; Chen, H. Preparation and adsorption properties of chelating resins containing 3-aminopyridine and hydrophilic spacer arm for $\mathrm{Hg}$ (II). Chem. Eng. J. 2010, 165, 573-580. [CrossRef]

41. Lei, P.; Lucy, C.A. Insight into the stability of poly(diallydimethylammoniumchloride) and polybrene poly cationic coatings in capillary electrophoresis. J. Chromatogr. A 2014, 1365, 226-233.

(C) 2019 by the authors. Licensee MDPI, Basel, Switzerland. This article is an open access article distributed under the terms and conditions of the Creative Commons Attribution (CC BY) license (http://creativecommons.org/licenses/by/4.0/). 